\title{
The SKA view of the Neutral Interstellar Medium in Galaxies
}

\author{
W.J.G. de Blok ${ }^{* a b c}$, F. Fraternali ${ }^{d c}$, G.H. Heald ${ }^{a c}$, E.A.K. Adams ${ }^{a}$, A. Bosma ${ }^{e}$, \\ Bärbel S. Koribalski ${ }^{f}$ and the $\mathrm{H}$ I Science Working Group \\ ${ }^{a}$ ASTRON \\ Postbus 2, 7990 AA, Dwingeloo, The Netherlands \\ ${ }^{b}$ Department of Astronomy, Astrophysics, Cosmology and Gravity Centre, \\ University of Cape Town, Private Bag X3, Rondebosch, 7701 South Africa \\ ${ }^{c}$ Kapteyn Astronomical Institute, University of Groningen \\ Postbus 800, 9700 AV, Groningen, The Netherlands \\ ${ }^{d}$ Department of Physics and Astronomy, University of Bologna, \\ via Berti Pichat 6/2, I-40127 Bologna, Italy \\ ${ }^{e}$ Aix Marseille Universite, CNRS, LAM (Laboratoire d'Astrophysique de Marseille) \\ UMR 7326, F-13388 Marseille, France \\ ${ }^{f}$ Australia Telescope National Facility, CSIRO Astronomy \& Space Science \\ P.O. Box 76, Epping, NSW 1710, Australia \\ E-mail: blokeastron.nl, filippo.fraternali@unibo.it, healdeastron.nl, \\ adams@astron.nl, bosma@lam.fr, Baerbel.Koribalski@csiro.au, \\ swg-higalaxyeskatelescope.org
}

\begin{abstract}
Two major questions in galaxy evolution are how star-formation on small scales leads to global scaling laws and how galaxies acquire sufficient gas to sustain their star formation rates. H I observations with high angular resolution and with sensitivity to very low column densities are some of the important observational ingredients that are currently still missing. Answers to these questions are necessary for a correct interpretation of observations of galaxy evolution in the high-redshift universe and will provide crucial input for the sub-grid physics in hydrodynamical simulations of galaxy evolutions. In this chapter we discuss the progress that will be made with the SKA using targeted observations of nearby individual disk and dwarf galaxies.
\end{abstract}

Advancing Astrophysics with the Square Kilometre Array

June 8-13, 2014

Giardini Naxos, Italy

\footnotetext{
* Speaker.
} 


\section{The Galactic Gas Cycle}

Galaxy evolution is driven by the flow of gas into galaxies, the transformation of gas into stars, and the expulsion of gas due to the subsequent stellar evolution processes. Atomic neutral hydrogen (H I) is an excellent tracer - and often the main constituent - of this gas, and can be observed readily in the $21-\mathrm{cm}$ line. The SKA will be able to directly trace the gradual transformation from primordial hydrogen into galaxies over cosmic time. However, to correctly interpret this evolution, direct detailed observations of the sub-kpc-scale physical processes that cause this transformation are essential for understanding the astrophysics of galaxy evolution.

Two very important processes in this cosmic evolution are star formation and gas accretion. The first one enriches the interstellar medium (ISM) with metals, injects energy into it, and, through the ejection of gas, also enriches the intergalactic medium (IGM). The second process delivers gas from outside galaxies (either primordial or previously ejected) into the star-forming disks, which ensures galaxies can keep forming stars over a Hubble time.

Star formation thus plays a central role in galaxy evolution, yet little is known about the necessary conditions for star formation to occur. Global, kpc-scale relations, such as the KennicuttSchmidt Law (Kennicutt 1989, 1998), have established that there is a direct connection between the (molecular) gas surface density and the star formation rate (SFR) surface density. This relation has been found to hold for both spiral galaxies, as well as late-type dwarf galaxies (see, e.g., Leroy et al. 2008 and Bigiel et al. 2008 for a recent analysis). How the underlying astrophysics at the scales of individual gas clouds and cloud complexes leads to such a relation on more global scales remains, however, ill-understood.

The initial phase of this star-formation sequence will presumably be the cooling of gas to temperatures below $\sim 10^{4} \mathrm{~K}$, where it can collapse and form clouds. Observing this cold, clumpy ISM and distinguishing it from the warmer, more diffuse phase requires imaging at high spatial and velocity resolution. To date, few $\mathrm{H}$ I observations of nearby galaxies resolve the individual cold gas complexes (see, e.g., Braun et al. 2009 and Kim et al. 2003 for examples in the Local Group). The SKA will have sufficient sensitivity at the required high resolution to do this for many galaxies. This enables studies of the properties of the cold gas in galaxies at the desired cloudsize resolution over a wide range of galaxy properties and environments. In addition, ALMA can observe the molecular components of these clouds and complexes, and this complementarity will lead to a revolutionary improvement in our understanding of star formation — and the conditions leading to it - in different environments.

A related question is: how can galaxies sustain their star formation over a Hubble time? In general, local galaxies only have enough gas to sustain their SFR for few Gyr, and they must thus acquire gas from somewhere else (see Sancisi et al. 2008 for a recent overview). Numerical simulations suggest gas flows in from the IGM (or "cosmic web"), through a process called "cold accretion" (e.g., Kereš et al. 2005). "Cold" in this context means that the gas has not been shockheated as it entered the galaxy halo. This cold accretion process is discussed in more detail in the chapter by Popping et al. (2015) elsewhere in this volume. So far there is little direct observational evidence for significant cold accretion. The observed cold gas accretion in galaxies seems to be an order of magnitude too low to explain the current star formation rates (SFR) in galaxies (Sancisi et al. 2008; Putman et al. 2012). If cold accretion is the dominant process by which galaxies acquire 
their gas, then current observational limits indicate it must happen at H I column densities below $\sim 10^{18} \mathrm{~cm}^{-2}$. Only a limited number of galaxies have so far been explored at sufficient sensitivity and spatial resolution to probe this regime (Heald et al. 2011; Putman et al. 2012). SKA will be able to directly detect and map in detail the gas at these column densities and trace its connection with the cosmic web.

The galactic fountain process (Shapiro \& Field 1976; Bregman 1980; Norman \& Ikeuchi 1989) links the gas in the star forming disk and that in the halo. Massive stars, through supernova explosions and stellar winds, can push gas out of the disk and into the halo of a galaxy. This creates the holes and bubbles frequently observed in the gas disks of galaxies (Bagetakos et al. 2011). The expelled gas will cool and eventually rain back on the disk, most likely in the form of $\mathrm{H}$ I clouds (Putman et al. 2012). Such clouds have also been observed in a number of other galaxies as part of an extra-planar gas component (Sancisi et al. 2008), and presumably form the equivalent of the high and intermediate velocity clouds (HVCs and IVCs, respectively) in our Galaxy. It is thought that the process of these clouds moving through the hot gaseous halo of a galaxy provides an alternative mechanism for accretion of gas. Here, hot halo gas cools in a cloud's wake and is dragged along as the cloud moves back into the disk (Fraternali 2013).

While the galactic fountain clouds thus provide a possible means for the transportation of gas into the disk, they also hinder our ability to identify primordial gas clouds that are being accreted from the IGM. A small number can be identified as they are counter-rotating with respect to their host galaxy (Oosterloo et al. 2007), but a better census down to lower H I masses and column densities is needed to properly understand these accretion mechanisms. Prime targets for this are galaxies in the 'Local Volume', defined as the sphere of radius $10 \mathrm{Mpc}$ centered on the Local Group (Karachentsev et al. 2004, 2013; Koribalski 2008). This volume includes around 900 galaxies, the majority of which are gas-rich. Distances to these galaxies are known with high accuracy. The H I contents and sizes of Local Volume galaxies cover more than two orders of magnitudes, ranging from low-mass dwarf galaxies with diameters of less than one kpc (e.g., Leo T; Ryan-Weber et al. 2008) to grand design spirals with H I diameters of $\sim 100 \mathrm{kpc}$ (e.g., Circinus; For et al. 2012).

In summary, the SKA will be a unique instrument that will be able to track the gas in lowredshift galaxies as it accretes onto the disk, forms $\mathrm{H}$ I cloud complexes (which in turn produce stars through an intermediate, molecular phase), is expelled through feedback processes, and subsequently returns to the disk. Complemented by ALMA molecular line observations, the transformation from neutral atomic and molecular gas to the formation of stars can thus be investigated in exceptional detail over a large range in galaxy properties (see, e.g., Ott et al. 2008 and Fukui et al. 2009 for work on the LMC and Stanimirović et al. 2014 for our Galaxy). These processes can be studied in detail in a variety of environments at low redshift, thus establishing the astrophysical foundations that are needed to interpret observations of galaxies at higher redshifts, and to guide the implementation of 'sub-grid' physics into hydrodynamical simulations of galaxy formation and evolution.

In the rest of this section we calculate the H I column density and mass sensitivities of SKA, SKA1-MID and SKA1-SUR for a number of observing scenarios. This chapter specifically deals with high-resolution and high-sensitivity observations of individual objects, i.e., deep observations of limited areas of sky.

The remainder of this chapter then discusses the prospects for obtaining detailed, sensitive 
observations of nearby, individual galaxies and the progress that will be made towards a better understanding of the link between star formation, accretion, and gas in these galaxies.

\subsection{SKA column density and $H$ I mass sensitivities}

We calculate column density sensitivities for SKA, SKA1-MID and SKA1-SUR for a range of angular and velocity resolutions and three representative integration times. For the latter we choose:

- 10 hours: representing a typical "single-track" observation (as motivated in Sect. 2.3). In terms of science, an observation like this can be used to characterise high-resolution structures in the star forming disks of galaxies, as well as (at lower spatial resolutions) the outer low-column density disk down to $N_{\mathrm{HI}} \sim 10^{18} \mathrm{~cm}^{-2}$;

- 100 hours: this longer observation will typically result in detection of $\mathrm{H} \mathrm{I} \mathrm{column} \mathrm{densities}$ down to $\sim 10^{17} \mathrm{~cm}^{-2}$ where accretion and faint outer gas can be directly studied;

- 1000 hours: this extremely long observation allows detection of column densities well below $10^{17} \mathrm{~cm}^{-2}$ where one can directly explore the link of galaxies with the cosmic web.

For the angular resolutions we choose representative beam sizes between $1^{\prime \prime}$ and $30^{\prime \prime}$. For the velocity resolution we choose values between 1 and $20 \mathrm{~km} \mathrm{~s}^{-1}$. Using these parameters we calculate the $5 \sigma$ column density detection limits per velocity channel. Our basis for this are the SKA1-MID and SKA1-SUR natural sensitivities as listed in Table 1 of the SKA Baseline Design Document (Dewdney et al. 2013). For SKA1-MID we use the sensitivity listed for 190 SKA dishes combined with the 64 MeerKAT dishes. For SKA1-SUR the sensitivity of 60 SKA dishes combined with 36 ASKAP dishes is used. For SKA1-MID the $1 \sigma$ sensitivity is given as $63 \mu \mathrm{Jy}_{\text {beam }}{ }^{-1}$ over $100 \mathrm{kHz}$ for one hour of observing time. For SKA1-SUR this value is $263 \mu \mathrm{Jy}^{\text {beam }}{ }^{-1}$ over 100 $\mathrm{kHz}$ after one hour. We scale these sensitivities using the appropriate integration time and velocity resolution. For the sensitivity scaling as a function of angular resolution we use the noise scaling curve shown in the bottom panel of Figure 1 of the SKA1 Imaging Science Performance document (Braun 2014). These scalings take into account the effect of the spatial tapering needed to achieve the desired resolution. In addition, we apply an overall efficiency factor of 0.9 as listed in Appendix A of Braun (2014). At 1.4 GHz SKA1-MID will have a field of view of 0.5 square degrees. SKA1SUR will be able to map 18 square degrees due to its wide-field phased array feeds.

The $5 \sigma$ column density limits for SKA1-MID, SKA1-SUR and SKA are given in Table 1. These values agree well with those listed in Popping et al. (2015) which were derived using simulations. Note that a column density limit is defined as the flux density limit times the velocity width, so for a fixed observation time and angular resolution, observing with a larger velocity channel width will lead to a higher column density limit. This is therefore distinctly different from the case where an already detected signal is smoothed to a larger channel width giving an improved sensitivity (but at the cost of a coarser velocity resolution).

For SKA, we assume that it has 10 times the natural sensitivity of SKA1-MID, and apply a $50 \%$ correction factor to take into account tapering and weighting. We assume this factor is independent of angular resolution.

In addition to column densities, we also list in Table 2 the limiting $\mathrm{H}$ I masses that can be detected using the various setups. Here we assume the sources are unresolved and have a top-hat velocity profile with a peak flux equal to $5 \sigma$ and a velocity width of $50 \mathrm{~km} \mathrm{~s}^{-1}$. Optimal smoothing 
(i.e., a channel width equal to the velocity width) is assumed. Mass limits for other velocity widths can be deduced by simply scaling with the width of the profile.

The science described here focuses on $\mathrm{H}$ I observations of low-redshift galaxies $(z<0.1)$ so only frequency band $2(950-1760 \mathrm{MHz}$ on SKA1-MID and 650-1760 MHz on SKA1-SUR) is needed.

\section{Gas, Star Formation and Dark Matter at High Resolution}

\subsection{Gas and Stars}

The transformation of gas into stars is one of the most important processes in galaxy evolution. Understanding the conditions that determine the efficiency of this process, and the associated physics, is the goal of many observational and theoretical studies. They also form important input into numerical models of galaxy formation and evolution. This requires knowledge of these processes over a large range in scales: from galaxy-sized scales where gas is transported from the disk of the galaxy into the halo and back, to kpc-sized scales where gas clouds are collapsing, via sub-kpc scales where neutral gas cools and turns molecular in Giant Molecular Clouds (GMC), to parsec scales where individual stars are formed. The latter, very small, scales can be directly observed in our Galaxy, while processes happening at galaxy scales can be studied in external galaxies. Tying together the processes happening at these two extreme scales is a major challenge: in our Galaxy we lack the overview, while in external galaxies we rarely have the required resolution and sensitivity to study these processes in detail.

Over the last decade the number of high-resolution, multi-wavelength studies of galaxies in the nearby universe has increased dramatically. The $\mathrm{H}$ I observations were obtained as part of dedicated surveys such as THINGS (Walter et al. 2008), Little THINGS (Hunter et al. 2012), VLA-ANGST (Ott et al. 2012), FIGGS (Begum et al. 2008), WHISP (van der Hulst et al. 2001), HALOGAS (Heald et al. 2011), SHIELD (Cannon et al. 2011), and LVHIS (Koribalski 2008). These surveys have made possible new studies of the conditions for star formation on kpc or even sub-kpc scales in a larger number of galaxies (see the chapter by Blyth et al. 2015 for a more extensive description of recent H I surveys). These have made it possible to make the first steps towards bridging the gap between the observations at the scales of stars and those at scales of galaxies. With the SKA the next step can be taken.

Kennicutt (1989) studied the gas and $\mathrm{H} \alpha$ content of a number of nearby galaxies and found a power-law relation between the total gas surface density and the $\mathrm{H} \alpha$-derived SFR. Over the years, similar studies (see e.g., Calzetti et al. 2012; Calzetti 2013), using different measures for the gas surface density, and different star formation tracers (such as ultra-violet, $\mathrm{H} \alpha$, infra-red or combinations of them) found similar relations, but with a large spread in the parameters. This may reflect actual variations in the physics, but a large part of this spread is very likely also due to choice of sample, analysis and star formation tracers.

Recent studies of the star-forming disks of nearby galaxies found a tight linear relation between the molecular gas surface density and the SFR surface density (Leroy et al. 2008; Bigiel et al. 2008). This can be interpreted as stars forming from the molecular ISM at a constant efficiency. Note though that these results do not say anything about the necessary conditions for star formation on 
Table 1: $5 \sigma \mathrm{H}$ I column density limits $\left(\mathrm{cm}^{-2}\right)$

\begin{tabular}{|c|c|c|c|c|}
\hline \multirow{2}{*}{$\begin{array}{l}\text { beam } \\
\text { size }\end{array}$} & \multicolumn{4}{|c|}{ velocity resolution } \\
\hline & $1 \mathrm{~km} \mathrm{~s}^{-1}$ & $5 \mathrm{~km} \mathrm{~s}^{-1}$ & $10 \mathrm{~km} \mathrm{~s}^{-1}$ & $20 \mathrm{~km} \mathrm{~s}^{-1}$ \\
\hline & \multicolumn{4}{|c|}{ SKA1-MID } \\
\hline \multicolumn{5}{|c|}{$t=10^{h}$} \\
\hline $1^{\prime \prime}$ & $1.75 \mathrm{E}+21$ & $3.91 \mathrm{E}+21$ & $5.53 \mathrm{E}+21$ & $7.83 \mathrm{E}+21$ \\
\hline $3^{\prime \prime}$ & $1.63 E+20$ & $3.65 \mathrm{E}+20$ & $5.16 \mathrm{E}+20$ & $7.30 \mathrm{E}+20$ \\
\hline $10^{\prime \prime}$ & $1.13 \mathrm{E}+19$ & $2.52 \mathrm{E}+19$ & $3.57 \mathrm{E}+19$ & $5.05 E+19$ \\
\hline $30^{\prime \prime}$ & $1.31 \mathrm{E}+18$ & $2.94 \mathrm{E}+18$ & $4.16 \mathrm{E}+18$ & $5.89 \mathrm{E}+18$ \\
\hline \multicolumn{5}{|c|}{$t=100^{h}$} \\
\hline $1^{\prime \prime}$ & $5.53 \mathrm{E}+20$ & $1.23 \mathrm{E}+21$ & $1.75 \mathrm{E}+21$ & $2.47 \mathrm{E}+21$ \\
\hline $3^{\prime \prime}$ & $5.16 \mathrm{E}+19$ & $1.15 \mathrm{E}+20$ & $1.63 \mathrm{E}+20$ & $2.30 \mathrm{E}+20$ \\
\hline $10^{\prime \prime}$ & $3.57 \mathrm{E}+18$ & $7.99 \mathrm{E}+18$ & $1.13 E+19$ & $1.59 \mathrm{E}+19$ \\
\hline $30^{\prime \prime}$ & $4.16 \mathrm{E}+17$ & $9.32 \mathrm{E}+17$ & $1.31 \mathrm{E}+18$ & $1.86 \mathrm{E}+18$ \\
\hline \multicolumn{5}{|c|}{$t=1000^{h}$} \\
\hline $1^{\prime \prime}$ & $1.75 \mathrm{E}+20$ & $3.91 \mathrm{E}+20$ & $5.53 \mathrm{E}+20$ & $7.83 \mathrm{E}+20$ \\
\hline $3^{\prime \prime}$ & $1.63 \mathrm{E}+19$ & $3.65 E+19$ & $5.16 \mathrm{E}+19$ & $7.30 \mathrm{E}+19$ \\
\hline $10^{\prime \prime}$ & $1.13 \mathrm{E}+18$ & $2.52 \mathrm{E}+18$ & $3.57 \mathrm{E}+18$ & $5.05 E+18$ \\
\hline \multirow[t]{2}{*}{$30^{\prime \prime}$} & $1.31 \mathrm{E}+17$ & $2.94 \mathrm{E}+17$ & $4.16 \mathrm{E}+17$ & $5.89 \mathrm{E}+17$ \\
\hline & \multicolumn{4}{|c|}{ SKA1-SUR } \\
\hline \multicolumn{5}{|c|}{$t=10^{h}$} \\
\hline $1^{\prime \prime}$ & $4.71 \mathrm{E}+21$ & $1.05 \mathrm{E}+22$ & $1.49 \mathrm{E}+22$ & $2.11 \mathrm{E}+22$ \\
\hline $3^{\prime \prime}$ & $4.45 \mathrm{E}+20$ & $9.96 \mathrm{E}+20$ & $1.40 \mathrm{E}+21$ & $1.99 \mathrm{E}+21$ \\
\hline $10^{\prime \prime}$ & $4.48 \mathrm{E}+19$ & $1.00 \mathrm{E}+20$ & $1.41 \mathrm{E}+20$ & $2.00 \mathrm{E}+20$ \\
\hline $30^{\prime \prime}$ & $8.38 \mathrm{E}+18$ & $1.87 \mathrm{E}+19$ & $2.65 E+19$ & $3.75 E+19$ \\
\hline \multicolumn{5}{|c|}{$t=100^{h}$} \\
\hline $1^{\prime \prime}$ & $1.49 \mathrm{E}+21$ & $3.33 \mathrm{E}+21$ & $4.71 \mathrm{E}+21$ & $6.67 \mathrm{E}+21$ \\
\hline $3^{\prime \prime}$ & $1.40 \mathrm{E}+20$ & $3.15 \mathrm{E}+20$ & $4.45 E+20$ & $6.30 \mathrm{E}+20$ \\
\hline $10^{\prime \prime}$ & $1.41 \mathrm{E}+19$ & $3.16 \mathrm{E}+19$ & $4.48 \mathrm{E}+19$ & $6.33 \mathrm{E}+19$ \\
\hline $30^{\prime \prime}$ & $2.65 \mathrm{E}+18$ & $5.93 \mathrm{E}+18$ & $8.38 \mathrm{E}+18$ & $1.18 \mathrm{E}+19$ \\
\hline \multicolumn{5}{|c|}{$t=1000^{h}$} \\
\hline $1^{\prime \prime}$ & $4.71 \mathrm{E}+20$ & $1.05 \mathrm{E}+21$ & $1.49 \mathrm{E}+21$ & $2.11 \mathrm{E}+21$ \\
\hline $3^{\prime \prime}$ & $4.45 E+19$ & $9.96 \mathrm{E}+19$ & $1.40 \mathrm{E}+20$ & $1.99 \mathrm{E}+20$ \\
\hline $10^{\prime \prime}$ & $4.48 \mathrm{E}+18$ & $1.00 \mathrm{E}+19$ & $1.41 \mathrm{E}+19$ & $2.00 \mathrm{E}+19$ \\
\hline \multirow[t]{2}{*}{$30^{\prime \prime}$} & $8.38 \mathrm{E}+17$ & $1.87 \mathrm{E}+18$ & $2.65 \mathrm{E}+18$ & $3.75 \mathrm{E}+18$ \\
\hline & \multicolumn{4}{|c|}{ SKA } \\
\hline \multicolumn{5}{|c|}{$t=10^{h}$} \\
\hline $1^{\prime \prime}$ & $1.13 \mathrm{E}+20$ & $2.52 \mathrm{E}+20$ & $3.57 \mathrm{E}+20$ & $5.05 \mathrm{E}+20$ \\
\hline $3^{\prime \prime}$ & $1.25 \mathrm{E}+19$ & $2.80 \mathrm{E}+19$ & $3.97 \mathrm{E}+19$ & $5.61 \mathrm{E}+19$ \\
\hline $10^{\prime \prime}$ & $1.13 \mathrm{E}+18$ & $2.52 \mathrm{E}+18$ & $3.57 \mathrm{E}+18$ & $5.05 \mathrm{E}+18$ \\
\hline $30^{\prime \prime}$ & $1.25 \mathrm{E}+17$ & $2.80 \mathrm{E}+17$ & $3.97 \mathrm{E}+17$ & $5.61 \mathrm{E}+17$ \\
\hline \multicolumn{5}{|c|}{$t=100^{h}$} \\
\hline $1^{\prime \prime}$ & $3.57 \mathrm{E}+19$ & $7.99 \mathrm{E}+19$ & $1.13 \mathrm{E}+20$ & $1.59 \mathrm{E}+20$ \\
\hline $3^{\prime \prime}$ & $3.97 \mathrm{E}+18$ & $8.87 \mathrm{E}+18$ & $1.25 \mathrm{E}+19$ & $1.77 \mathrm{E}+19$ \\
\hline $10^{\prime \prime}$ & $3.57 \mathrm{E}+17$ & $7.99 \mathrm{E}+17$ & $1.13 \mathrm{E}+18$ & $1.59 \mathrm{E}+18$ \\
\hline $30^{\prime \prime}$ & $3.97 \mathrm{E}+16$ & $8.87 \mathrm{E}+16$ & $1.25 \mathrm{E}+17$ & $1.77 \mathrm{E}+17$ \\
\hline \multicolumn{5}{|c|}{$t=1000^{h}$} \\
\hline $1^{\prime \prime}$ & $1.13 \mathrm{E}+19$ & $2.52 \mathrm{E}+19$ & $3.57 \mathrm{E}+19$ & $5.05 \mathrm{E}+19$ \\
\hline $3^{\prime \prime}$ & $1.25 \mathrm{E}+18$ & $2.80 \mathrm{E}+18$ & $3.97 \mathrm{E}+18$ & $5.61 \mathrm{E}+18$ \\
\hline $10^{\prime \prime}$ & $1.13 \mathrm{E}+17$ & $2.52 \mathrm{E}+17$ & $3.57 \mathrm{E}+17$ & $5.05 \mathrm{E}+17$ \\
\hline $30^{\prime \prime}$ & $1.25 \mathrm{E}+16$ & $2.80 \mathrm{E}+16$ & $3.97 \mathrm{E}+16$ & $5.61 \mathrm{E}+16$ \\
\hline
\end{tabular}


Table 2: H I mass limits for unresolved sources

\begin{tabular}{|c|c|c|c|c|c|c|}
\hline \multirow[t]{2}{*}{ beam } & \multicolumn{3}{|c|}{$M_{\mathrm{HI}} / D_{\mathrm{Mpc}}^{2}$ for SKA1-MID } & \multicolumn{3}{|c|}{$M_{\mathrm{HI}} / D_{\mathrm{Mpc}}^{2}$ for SKA1-SUR } \\
\hline & $t=10^{h}$ & $t=100^{h}$ & $t=1000^{h}$ & $t=10^{h}$ & $t=100^{h}$ & $t=1000^{h}$ \\
\hline $1^{\prime \prime}$ & $2.6 \mathrm{E}+03$ & $8.3 \mathrm{E}+02$ & $2.6 \mathrm{E}+02$ & $7.1 \mathrm{E}+03$ & $2.2 \mathrm{E}+03$ & $7.1 \mathrm{E}+02$ \\
\hline $3^{\prime \prime}$ & $2.1 \mathrm{E}+03$ & $7.0 \mathrm{E}+02$ & $2.2 \mathrm{E}+02$ & $6.0 \mathrm{E}+03$ & $1.9 \mathrm{E}+03$ & $6.0 \mathrm{E}+02$ \\
\hline $10^{\prime \prime}$ & $1.7 \mathrm{E}+03$ & $5.3 \mathrm{E}+02$ & $1.7 \mathrm{E}+02$ & $6.7 \mathrm{E}+03$ & $2.1 \mathrm{E}+03$ & $6.7 \mathrm{E}+02$ \\
\hline $30^{\prime \prime}$ & $1.8 \mathrm{E}+03$ & $5.6 \mathrm{E}+02$ & $1.8 \mathrm{E}+02$ & $1.1 \mathrm{E}+04$ & $3.6 \mathrm{E}+03$ & $1.1 \mathrm{E}+03$ \\
\hline
\end{tabular}

the scales of GMCs or smaller. With the analysis performed at a resolution of $750 \mathrm{pc}$, any relation must be interpreted within the context of "counting clouds", i.e., one has to assume that the GMCs have uniform properties with the observed molecular surface density determined by the beam filling factor of these clouds. Higher resolutions are needed to go beyond this limitation.

Schruba et al. (2011) found that the linear relation between molecular gas and SFR surface densities extends into the $\mathrm{H}$ I-dominated regime of the outer disks of galaxies (see also Roychowdhury et al. 2014). This is supported by the realisation that star formation in the outer parts of disks is more widespread than originally thought. GALEX UV observations enabled direct detection of $\mathrm{O}$ and $\mathrm{B}$ stars which would otherwise have escaped detection due to their inability to excite the surrounding ISM enough to produce $\mathrm{H} \alpha$ emission. It is now thought that these extended UV (XUV) disks are found in $\sim 30 \%$ of nearby disk galaxies (Thilker et al. 2007). A striking example is M83 (NGC 5236) as shown in Fig. 1. The outlying H I structures show up remarkably well in the UV, indicating that star formation is progressing there as well, albeit with a lower efficiency (Bigiel et al. 2011) than in the bright, inner region of the galaxy. An extreme example of this is the dwarf galaxy ESO215-G?009 (Warren et al. 2004). This galaxy has the highest gas-to-light ratio known for a galaxy in the low-redshift universe, yet, despite the large gas reservoir, star formation apparently has halted, is inhibited or lacks a trigger.

The above broad-brush picture illustrates the progress made in the last few years, and mentions some of the empirical relations that are now routinely used as input for numerical models that attempt to explain the variations of star formation efficiency with, e.g., redshift, environment or galaxy mass. It is however still difficult to link the observations with the actual physical processes driving the star formation rate. For example, Leroy et al. (2008) test a number of theoretical explanations proposed in the literature linking the gas density and the SFR. They looked at the disk free fall time, the orbital timescale, the effects of cloud-cloud collisions, the assumption of fixed GMC star formation efficiency, and the relation between pressure in the ISM and the phases of the ISM. Their conclusions were that none of these offers a unique explanation for the observed behavior. So, though large-scale relations can be identified (such as the Kennicutt-Schmidt Law), the actual physics is a lot more complicated, and happens below the resolutions achieved so far. Observationally, we will therefore have to probe the ISM at scales below that of GMCs and individual H I complexes. We also need to have a better understanding of the balance between warm and cold $\mathrm{H} \mathrm{I}$ phases, the efficiency of $\mathrm{H}_{2}$ formation and the effects of shocks and turbulence. Examples of the importance of high resolution and high sensitivity for the study of turbulence in $\mathrm{H}$ I are found in, e.g., Dutta et al. (2009, 2013). 


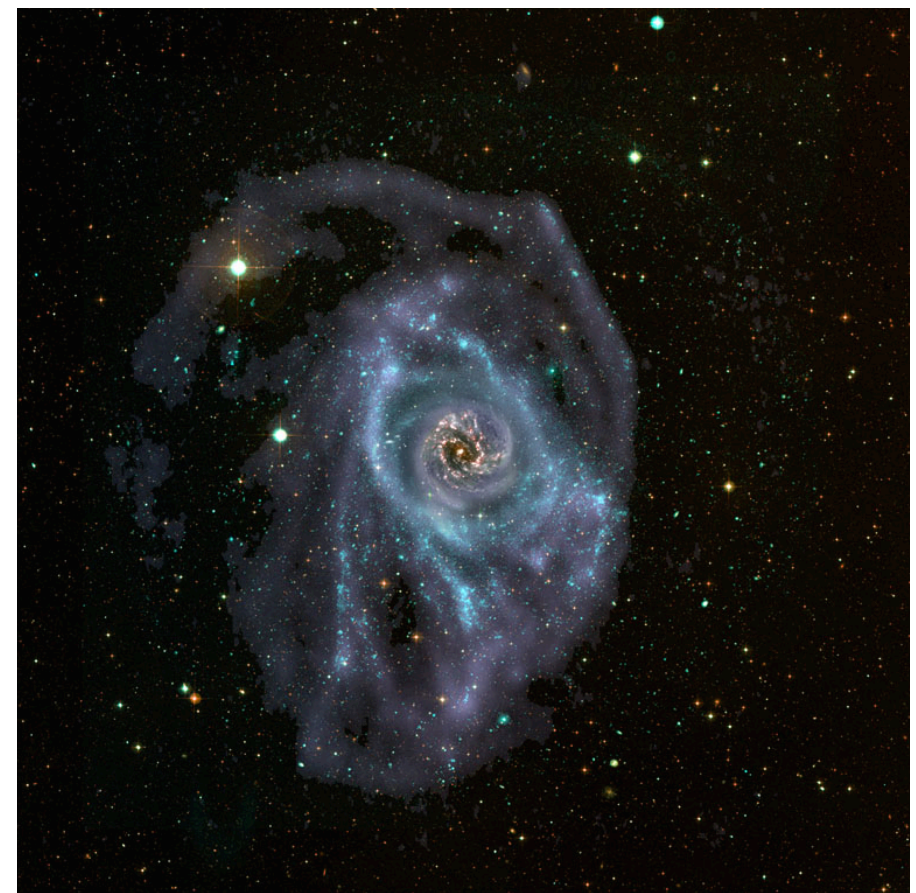

Figure 1: Composite multi-wavelength image of M83. Near- and far-UV from GALEX are shown in blue. Optical $R$-band from SDSS in green, $J$-band from 2 MASS in red, H I data from LVHIS is shown in paleblue, and a combination of $B, R$ and $\mathrm{H} \alpha$ have been used as the luminosity of the stellar disk. The field of view is $1.3^{\circ} \times 1.5^{\circ}$. (Picture courtesy B. Koribalski)

Young \& Lo (1996, 1997), Young et al. (2003), de Blok \& Walter (2006), Ianjamasimanana et al. (2012), Warren et al. (2012) and Stilp et al. (2013) all analyzed the H I emission velocity profiles of galaxies and found evidence for the presence of cold and warm $\mathrm{H} \mathrm{I}$ components by decomposing the velocity profiles into components with a high and a low velocity dispersion. They found that the cold (low velocity dispersion) component is usually located near star-forming regions, whereas the warm (high velocity dispersion) component tends to be found along every line of sight. This presumably tells us something about the conditions for the warm $\mathrm{H}$ I to cool and turn molecular, but the key limitation of these studies was again spatial resolution. The improved capabilities of the SKA will enable these studies to be repeated but now resolving the individual gas complexes. (Equivalent absorption line studies are described in the chapters by Morganti et al. 2015 and McClure-Griffiths et al. 2015.) This should thus provide observations of a large number of galaxies at sufficient resolution to gauge the ability of and the conditions for the ISM to form GMCs over a wide range of galaxy conditions and environments.

An angular resolution of $1^{\prime \prime}$ allows $100 \mathrm{pc}$ physical resolutions out to $20 \mathrm{Mpc}$ (i.e., the distance of the Virgo cluster). The high-resolution observations of M31 by Braun et al. (2009) (shown in Fig. 2) have a maximum resolution of 50 pc, while the LMC observations by Kim et al. (2003) probe scales of $15 \mathrm{pc}$. Assuming SKA can observe efficiently up to $\delta \sim+35^{\circ}$, then gives access to $\sim 800$ galaxies within $20 \mathrm{Mpc}$ that have independent distance measurements. Of these $\sim 80 \%$ have $\mathrm{H}$ I detections. With the SKA these can therefore all be studied at a resolution close to that of the Braun et al. (2009) M31 observations. Out to $3 \mathrm{Mpc}$, we can study galaxies at the same resolution 


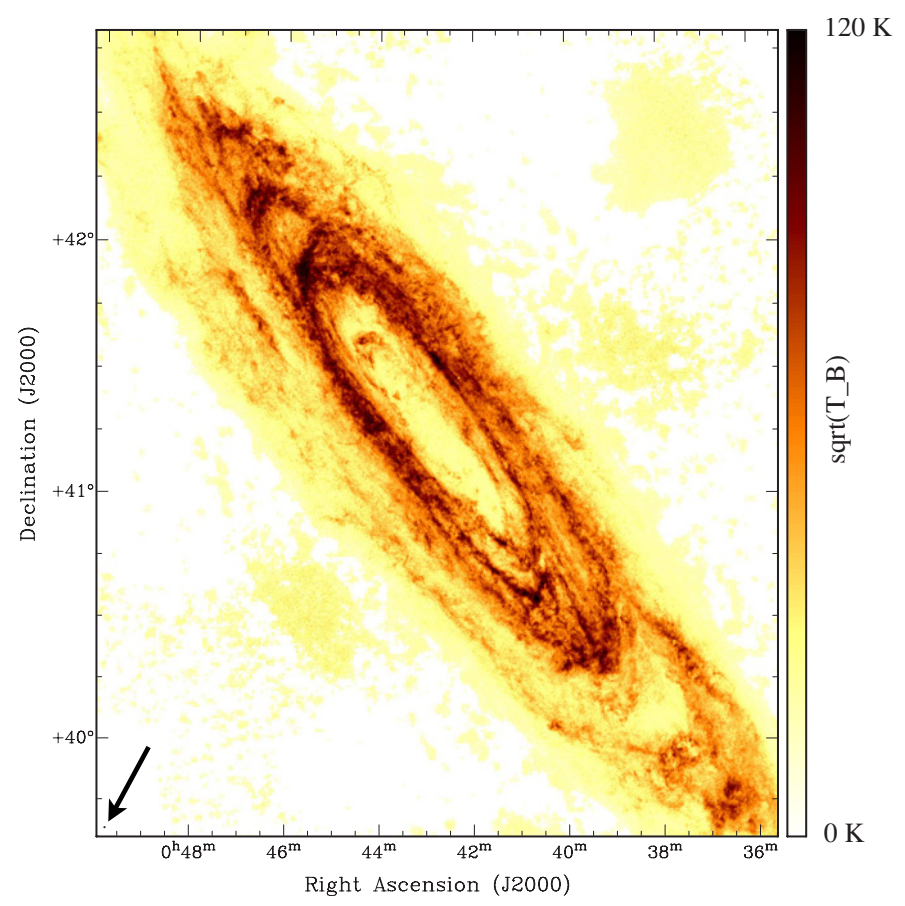

Figure 2: Integrated H I map of M31 from high-resolution observations by Braun et al. (2009). The resolution of these observations is $\sim 50 \mathrm{pc}$. The beam is indicated by the arrow in the bottom-left corner. SKA will enable highly detailed observations of this kind for hundreds of galaxies.

of the Kim et al. (2003) LMC observations. There are $\sim 75$ galaxies with independent distance measurements within that distance in the part of the sky accessible to the SKA. This includes, for example, the Sculptor group.

There are therefore a significant number of galaxies out to these distances, and this opens up the exciting prospect of being able to characterize the properties and morphologies of individual $\mathrm{H}$ I clouds in other galaxies over a wide range of environments. Combination with high-resolution ALMA observations should provide a comprehensive picture of the condition for and first phases of star formation.

\subsection{Dynamics and Dark Matter}

The high angular resolution that can be achieved with SKA will also be of importance to studies of the internal dynamics of galaxies and the distribution of dark matter.

H I studies played a major role in the 1970s and 1980s to establish the presence of dark matter in gas rich galaxies, necessary to keep up the extended, flat (or rising) rotation curves at large radii. More recent work on late-type low surface brightness galaxies showed that pure $\Lambda$ CDM models are in disagreement with observations, the so-called core-cusp controversy (de Blok 2010). Recent attempts to solve this problem concentrate on introducing feedback in the star formation recipes used in the numerical simulations of galaxy formation (Oh et al. 2011), so that an initially cuspy dark matter distribution can be modified sufficiently. Research on this problem can be expected to continue to progress, until a better understanding of these feedback mechanisms has been reached. At present, the predictions from these simulations seem to begin to work for small galaxies, but for 
larger ones the answer is still open. This is an active area of research, and further improvements of the galaxy formation models can be expected (e.g., di Cintio et al. 2014).

In terms of further $\mathrm{H}$ I observations addressing this problem, surveys planned with the SKA precursors will enable the selection of an adequate sample of relatively unperturbed galaxies, for which far deeper $\mathrm{H}$ I observations can be done with the SKA, as well as molecular gas observations with ALMA. These data will yield detailed information on the circular and (equally important) non-circular motions in these galaxies. Combined with more extensive diagnostics of the stellar kinematics using optical integral-field units, this will yield crucial information about the galaxy kinematics, and hence can be used to study the dark matter problem. Moreover, they can also be used for the purposes of studying the star formation and gas accretion in disk galaxies, as discussed in this chapter.

\subsection{SKA Prospects}

Here we use the column density limits listed in Table 1 and explore how the increased resolution and sensitivity of SKA makes new science possible. We use the results from the THINGS survey (Walter et al. 2008) as a benchmark. THINGS observed 34 nearby disk galaxies with the combined VLA B, C and D array with a maximum angular resolution of $6^{\prime \prime}$, or $\sim 500 \mathrm{pc}$ on average. For each galaxy, observing time was $7^{h}$ with the VLA B-array, $2.5^{h}$ with the C-array and $1.5^{h}$ with the D-array. A total of $\sim 10^{h}$ is therefore needed in this setup to produce a complete observation - this motivates our choice for $10^{h}$ as a typical observing time in Sect. 1.1. With these parameters, the column density sensitivity of the THINGS observations is $2.7 \cdot 10^{20} \mathrm{~cm}^{-2}$ for a $5 \sigma$ detection over a $5 \mathrm{~km} \mathrm{~s}^{-1}$ channel at $6^{\prime \prime}$ resolution (Walter et al. 2008). This angular resolution is effectively also the highest that can be achieved with the VLA B-array at $1.4 \mathrm{GHz}$, and is currently the highest resolution at which $\mathrm{H}$ I can still be observed routinely.

To compare prospective SKA observations directly with THINGS, we focus here on limits derived with a channel width of $5 \mathrm{~km} \mathrm{~s}^{-1}$. A $10^{h}$ integration time at $6^{\prime \prime}$ with SKA1-MID will achieve a column density limit of $8.1 \cdot 10^{19} \mathrm{~cm}^{-2}$. This is close to the $4 \cdot 10^{19} \mathrm{~cm}^{-2}$ THINGS sensitivity at $30^{\prime \prime}$. A $10^{h}$ observation on SKA1-MID at $6^{\prime \prime}$ thus enables studies at the resolution of THINGS, but to column density limits that are a factor of 3 deeper, thus probing the $\mathrm{H}$ I well outside the star forming disks. The larger sensitivity of SKA1-MID allows tapering to spatial resolutions higher than used by THINGS. For $10^{h}$ and at $3^{\prime \prime}$, the sensitivity at is $3.7 \cdot 10^{20} \mathrm{~cm}^{-2}$. This is comparable to the THINGS sensitivity at $6^{\prime \prime}$ and therefore allows study of the star-forming disk at twice the spatial resolution. For galaxies out to $10 \mathrm{Mpc}$ the linear resolution corresponding to $3^{\prime \prime}$ is better than $\sim 145 \mathrm{pc}$, which is starting to resolve individual $\mathrm{H}$ I complexes.

With the current baseline design, $3^{\prime \prime}$ is an upper limit in terms of resolution on SKA1-MID. There is not enough sensitivity on the longer baselines to push the resolution higher. For example, performing the same $10^{h}$ observation described above at $1^{\prime \prime}$ gives a column density limit of $3.9 \cdot 10^{21}$ $\mathrm{cm}^{-2}$, which is higher than the maximum face-on column densities typically found in galaxies at scales of hundreds of parsecs $\left(\sim 10^{21} \mathrm{~cm}^{-2}\right.$; Bigiel et al. 2008). It is possible that optical depth effects mask higher column densities at much smaller scales (e.g., Braun et al. 2009), but this has been tested only in a limited number of galaxies, and is the kind of project one would tackle with the SKA for a larger sample. 
Using SKA1-MID to make a longer observation of $100^{h}$, we reach column density limits of $1.2 \cdot 10^{20} \mathrm{~cm}^{-2}$ at $3^{\prime \prime}$ resolution (again using a channel spacing of $5 \mathrm{~km} \mathrm{~s}^{-1}$ ). This is a factor of $\sim 2-3$ deeper than THINGS at twice the spatial resolution, and will enable high-resolution characterization of the ISM at scales better than $145 \mathrm{pc}$ out to $10 \mathrm{Mpc}$.

Even with an increased observing time, resolutions of $1^{\prime \prime}$ remain challenging. At $100^{h}$ the limit is $1.2 \cdot 10^{21} \mathrm{~cm}^{-2}$ which is comparable to the highest column densities typically found in galaxies. At 1000 hours, the corresponding limit is around $3.9 \cdot 10^{20} \mathrm{~cm}^{-2}$, and this would allow mapping of most of the $\mathrm{H}$ I disk. This seems, however, a large investment of time for a single galaxy. For high-resolution observations of $\mathrm{H}$ I in nearby galaxies using SKA1-MID, $3^{\prime \prime}$ thus seems to be the practical (design-imposed) limit.

For a 50\% SKA1-MID, many aspects of the science mentioned above can still be done. The noise will be a factor two higher, but this still allows $3^{\prime \prime}$ resolution observations that, even at $10^{h}$, lead to sensitivities that are better than THINGS at $6^{\prime \prime}$.

At $10^{h}$ observing time, the $5 \sigma$ limits for SKA1-SUR at resolutions better than $5^{\prime \prime}$ are too high for mapping galaxies. For resolutions between $\sim 10^{\prime \prime}$ and $\sim 30^{\prime \prime}$ the sensitivities are high enough to perform THINGS-like observations. The strength of the SKA1-SUR observations will, however, be in their ability to characterize the environment due to its large instantaneous field-of-view of 18 square degrees.

The increased sensitivity of a full SKA means that an order of magnitude increase in column density sensitivity can be achieved with respect to SKA1-MID. A $10^{h}$ observation will reach down to $2.8 \cdot 10^{19} \mathrm{~cm}^{-2}$ at $3^{\prime \prime}$ and $5 \mathrm{~km} \mathrm{~s}^{-1}$ resolution. At $1^{\prime \prime}$ and $5 \mathrm{~km} \mathrm{~s}^{-1}$ resolution, the column density sensitivity that is achieved is comparable to that of THINGS (at $6^{\prime \prime}$ ), but with a spatial resolution that is a factor of 6 better.

At $100^{h}, 3^{\prime \prime}$ observations with SKA reach levels around $8.9 \cdot 10^{18} \mathrm{~cm}^{-2}$, with even a $1^{\prime \prime}$ beam giving limits of $8.0 \cdot 10^{19} \mathrm{~cm}^{-2}$, i.e., enough sensitivity to reach the levels typically found in the outer disks of galaxies. The Braun et al. (2009) observations of M31 (Fig. 2) have a linear resolution of $50 \mathrm{pc}$. With SKA at $1^{\prime \prime}$ we can thus reach similar resolutions out to $10 \mathrm{Mpc}$. This opens the exciting prospect of mapping galaxies at a physical resolution comparable to the M31 observations shown in Fig. 2. Furthermore, the SKA would make it possible to do this for hundreds of galaxies. Characterisation of the ISM at a resolution of tens of parsecs in such a large number of galaxies, each presumably with different physical conditions, would dramatically improve our knowledge of the ISM in other galaxies.

\section{The Galactic Fountain and Accretion}

\subsection{Background}

Galaxies are not isolated systems, as they continuously interact and exchange material with the environment in which they reside. Understanding how and in what form gas is transferred to galaxies from the IGM and vice-versa is a major challenge for current cosmological models. Starforming galaxies are expected to accrete fresh gas to feed their star formation throughout the whole Hubble time. Evidence for gas accretion includes estimates of the gas depletion times (Bigiel et al. 2011), the study of the star formation histories (Panter et al. 2007) and chemical evolution models 


\section{Neutral gas around galaxies: NGC891}

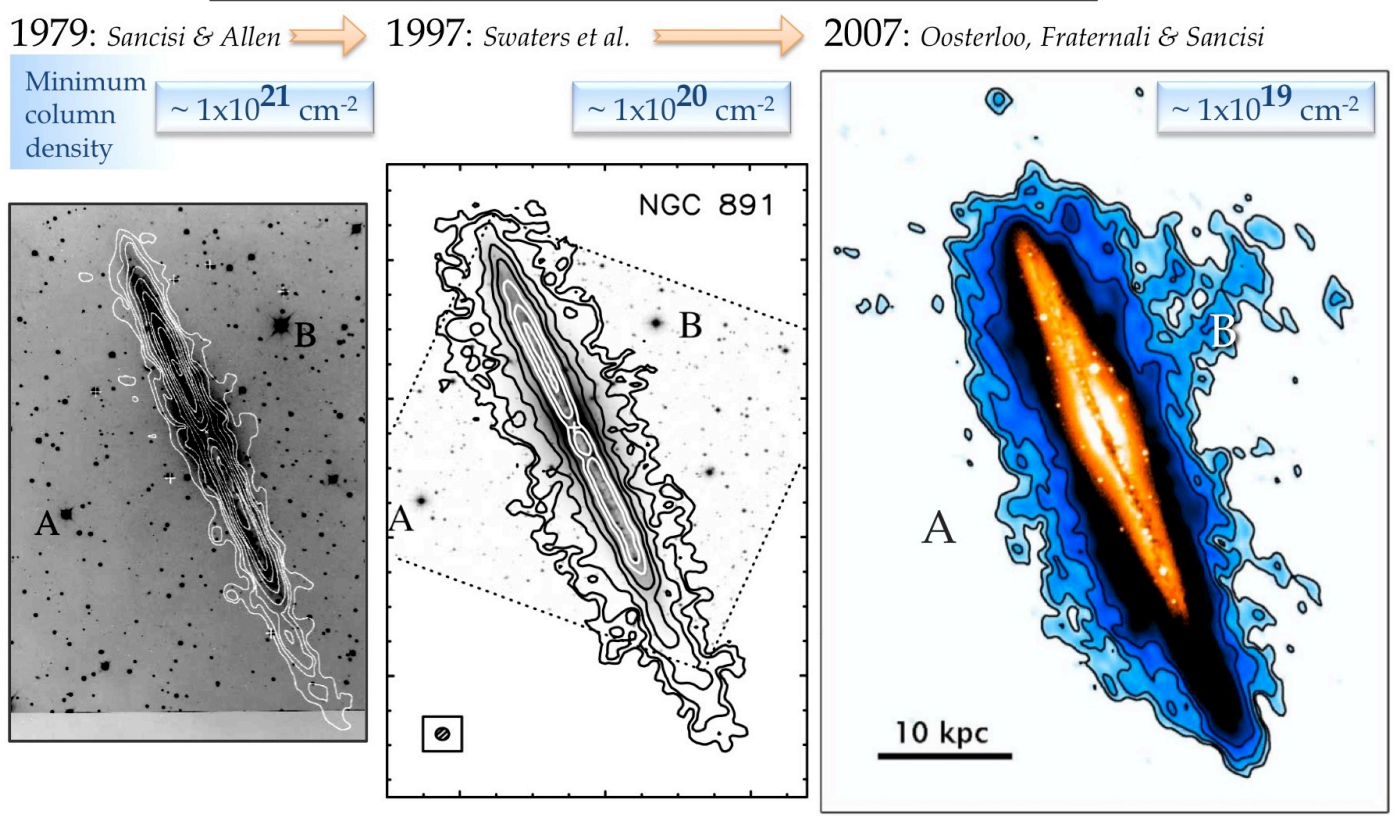

Figure 3: Three generations of neutral hydrogen observations of the edge-on spiral galaxy NGC 891, obtained with the Westerbork Synthesis Radio Telescope in 1979 (Sancisi \& Allen 1979) (left panel), 1997 (Swaters et al. 1997) (middle panel) and 2007 (Oosterloo et al. 2007) (right panel). The improvement in sensitivity of only one order of magnitude between each observation led to the discovery of the $\mathrm{H}$ I halo (middle panel) and next to the realization that this halo comprises almost $30 \%$ of the $\mathrm{H}$ I mass of the gas and hosts gas filaments extending up to more than $20 \mathrm{kpc}$ from the disk (right panel). SKA will go down two more orders of magnitude in column density, we can only wonder what we will find.

of the Milky Way (Chiappini et al. 1997; Schönrich \& Binney 2009). Indirect determinations of the gas accretion rate show that it should closely follow the SFR in every galaxy (Hopkins et al. 2008; Fraternali \& Tomassetti 2012). However, there is little evidence for the majority of the gas accretion taking place in the form of gas clouds infalling into local galaxies. In the Milky Way, the accretion rate from high velocity clouds is only $0.08 M_{\odot} \mathrm{yr}^{-1}$ (Putman et al. 2012), more than an order of magnitude lower than the SFR $\left(1-3 M_{\odot} \mathrm{yr}^{-1}\right.$, Chmoiuk \& Povich 2011). H I studies of nearby galaxies give similar discrepancies (Sancisi et al. 2008).

A possibility is that accretion takes place at very low column densities. At the moment the deepest $\mathrm{H}$ I observations of nearby disk galaxies obtained with an interferometer reach column densities slightly below $10^{19} \mathrm{~cm}^{-2}$ (Oosterloo et al. 2007; Walter et al. 2008; Westmeier et al. 2011; Heald et al. 2011). Fig. 3 shows three observations of the same galaxy, NGC 891, obtained in the last thirty years with the Westerbork Synthesis Radio Telescope (WSRT). The improvement in sensitivity from $\sim 1 \cdot 10^{21} \mathrm{~cm}^{-2}$ (left panel; Sancisi \& Allen 1979) to $\sim 1 \cdot 10^{19} \mathrm{~cm}^{-2}$ (right panel; Oosterloo et al. 2007) has revealed a completely different picture of the H I distribution in this galaxy. The most recent data have shown the presence of an extended $\mathrm{H}$ I halo which everywhere extends up to $8-10 \mathrm{kpc}$ above the disk of NGC 891 , and with a long filament extending up to $20 \mathrm{kpc}$. Most of this gas is likely to be produced by stellar feedback from the disk (Fraternali \& 
Binney 2008) but a fraction could come from accretion. The long filament is, however, likely due to a minor interaction (cf. Oosterloo et al. 2007; Mapelli et al. 2008). Clouds and filaments at similar column densities are observed also around other galaxies (Fraternali et al. 2002; Mundell et al. 2004; Westmeier et al. 2005; Sancisi et al. 2008). As an aside, a similar increase in the prominence of $\mathrm{HI}$ when going to lower column densities is also found for early-type galaxies. Here the $\mathrm{H} \mathrm{I}$ detection rate outside clusters increases from $\sim 0 \%$ to $\sim 40 \%$ when going from $1 \cdot 10^{21} \mathrm{~cm}^{-2}$ to $1 \cdot 10^{19} \mathrm{~cm}^{-2}$ (Serra et al. 2012).

Gas at lower column densities is therefore certainly present around galaxies. This is indicated by deep single-dish observations (e.g., de Blok et al. 2014), and by the study of Lyman- $\alpha$ absorbers towards quasars and their association with the circumgalactic media of nearby galaxies (Tumlinson et al. 2013). The distribution of this ultra-low column density gas can only be directly measured by the SKA.

The presence of low-column density gas around galaxies is also predicted by models. For example, some recent cosmological simulations show cold gas filaments penetrating the hot halos of galaxies and reaching the disks to feed star formation (e.g., Fernandez et al. 2012), though the magnitude of the implied accretion is still a matter of some debate (Nelson et al. 2013). Finally, galactic fountain models predict that the hot gaseous halos of galaxies are polluted and cooled by stellar feedback from the galactic disks (Fraternali \& Binney 2008; Marinacci et al. 2010). All these models make predictions on the presence and distribution of low column density material that can be directly tested by SKA (see also the chapter by Popping et al. 2015).

SKA will as well reveal the fate of the "missing" $\mathrm{H}$ I in more high-density environments such as Hickson Compact Groups (Verdes-Montenegro et al. 2001; Borthakur et al. 2010), which is suspected to be in the form of low-column density intra-group material extending to large relative velocities.

Given the dramatic difference which two orders of magnitude make, as illustrated in Fig. 3, it is difficult to anticipate what the full SKA will be able to see when going down to a sensitivity of below $10^{17} \mathrm{~cm}^{-2}$. However, what is clear, is that with SKA we will obtain the full picture of $\mathrm{H} \mathrm{I}$ around galaxies, characterize the properties and the kinematics of the $\mathrm{H}$ I halos, and derive precise estimates of gas accretion. SKA will provide a definitive answer on how gas falls into galaxies from intergalactic space.

\subsection{Current Status}

The WSRT Hydrogen Accretion in Local Galaxies (HALOGAS) survey (Heald et al. 2011) aims to catalogue accreting gas in nearby spiral galaxies, and to determine the ubiquity, properties, and origin of neutral gas halos such as the one associated with NGC 891 (Fig. 3). The HALOGAS survey observed 24 galaxies for 120 hours each to a column density limit of $\sim 1 \cdot 10^{19} \mathrm{~cm}^{-2}$ (assuming a $5 \sigma$ limit and a linewidth of $12 \mathrm{~km} \mathrm{~s}^{-1}$ ). As noted above, this level is suitable for confidently detecting and characterizing diffuse neutral extraplanar gas, and also for identifying isolated clouds around the target galaxies. The HALOGAS detection limit for unresolved cloud masses is approximately $M_{\mathrm{HI}} \approx 2.7 \cdot 10^{5}(D / 10 \mathrm{Mpc})^{2} M_{\odot}$. The HALOGAS observations with the WSRT attain the best compromise between angular resolution and sensitivity at around $30^{\prime \prime}$, meaning that for a galaxy at a typical distance of $10 \mathrm{Mpc}$, the linear resolution is around $1.5 \mathrm{kpc}$. While a higher resolution survey like THINGS is better suited for study of small-scale structure in the ISM and the 

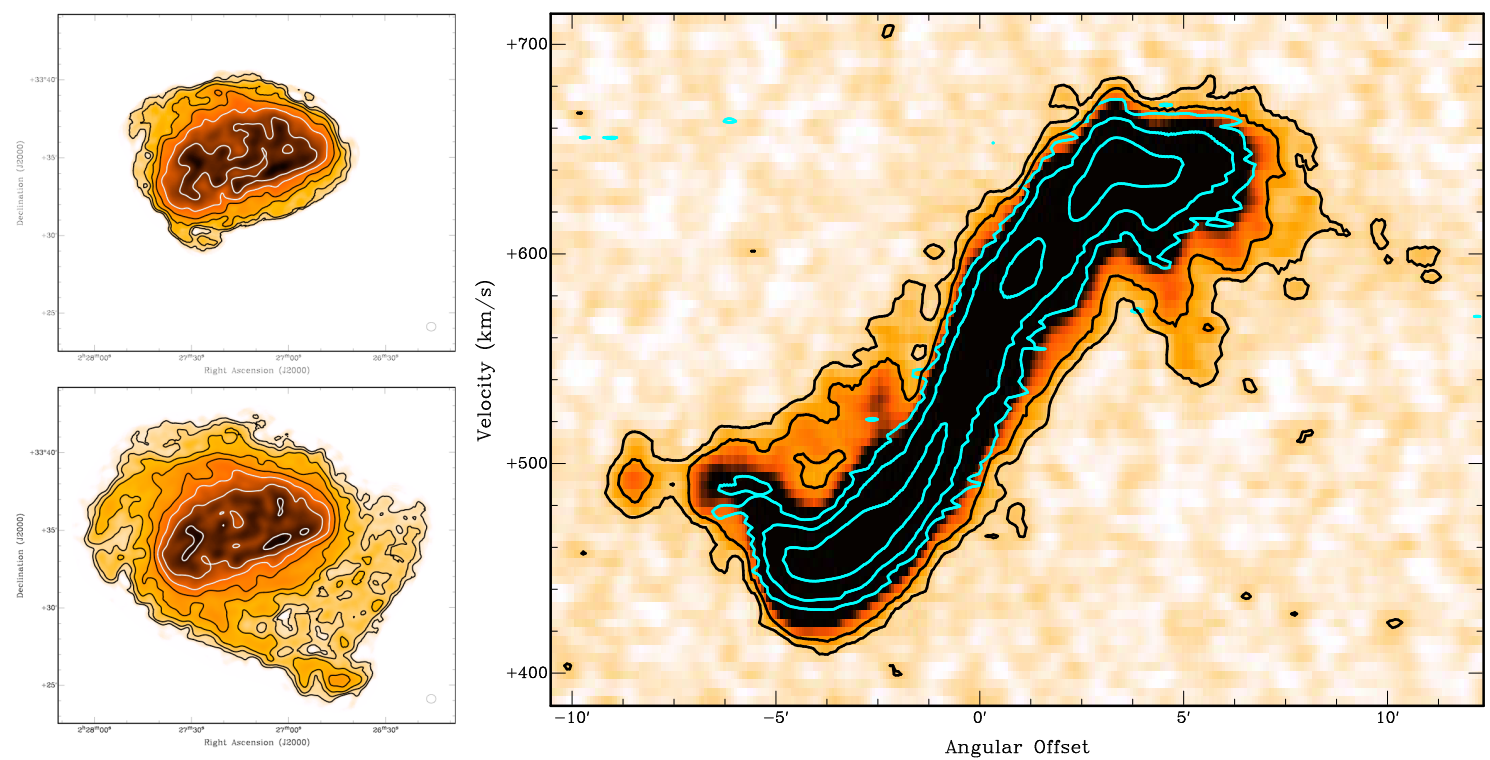

Figure 4: Comparison between THINGS and HALOGAS data for NGC 925. The THINGS data have been smoothed to the same resolution $\left(\sim 30^{\prime \prime}\right)$ as the HALOGAS data. Top left: THINGS moment- 0 . The lowest contour is at $5 \cdot 10^{19} \mathrm{~cm}^{-2}$, and contour levels increase by factors of two. Bottom left: HALOGAS moment0 ; the lowest contour is $1 \cdot 10^{19}$ and levels increase by a ctor of two. Right: PV diagram near the major axis (grayscale and black contours from HALOGAS at 3,9 $\sigma$, cyan from THINGS at 3,9,27 $\sigma$ ). This figure shows how the increased column density sensitivity of HALOGAS enables mapping of the low column density gas in and around galaxies.

connection to star formation (as discussed in the previous section), the HALOGAS observations are much more sensitive to diffuse structures (see as an example data for NGC 925 in Fig. 4).

HALOGAS provides the capability for a uniquely deep and uniform search for gas clouds and streams in the environment of the target galaxies. These clouds and streams, if present, could represent the most obvious signposts of ongoing cold accretion in the local Universe. A complete accounting of the potentially accreting gas is still being finalized (Jütte et al. 2015) but it can already be concluded that the total visible accretion rate attributable to such features is rather small. HALOGAS shows that a substantial population of gas clouds and streams in neutral gas is not present down to the $\sim 10^{19} \mathrm{~cm}^{-2}$ survey column density limit. There are clear consequences for the mechanisms that can be invoked to explain the continued fuelling of star formation and the column density levels at which these occur.

Crucially, the benefit of deep observations is not just the sensitivity to faint diffuse outer disk emission, but also to faint diffuse thick disk gas seen in projection against the inner thin (main) disk. Analysis of the HALOGAS sample shows that the key discriminating factor in determining the presence of such a thick $\mathrm{H}$ I disk is the star formation rate normalized by the disk area (Heald et al. 2015). These thick disks in many cases show rotation speeds which are smaller than those in the plane of the disk (e.g., Oosterloo et al. 2007). The dependence on star formation rate surface density points strongly toward the galactic fountain being the dominant factor in creating thick H I disks in galaxies. It looks consistent with the picture of galactic-fountain-driven positive feedback (Fraternali \& Binney 2008; Marinacci et al. 2010). The presence and importance 
of extra-planar material (such as a thick H I disk) would then be the relevant observational tracer of the gas accretion process for galaxies in the local Universe. This result bears confirmation and further investigation with the SKA.

\subsection{SKA Prospects}

The current configuration of SKA1-MID is heavily dominated by short baselines and therefore has an exquisite column density sensitivity at moderate resolutions. To compare its performance with existing observations we use the HALOGAS survey (Heald et al. 2011) as described above as a benchmark. HALOGAS reaches a typical $5 \sigma$ column density sensitivity of $5 \cdot 10^{18} \mathrm{~cm}^{-2}$ per $4.1 \mathrm{~km} \mathrm{~s}^{-1}$ channel at a spatial resolution of $30^{\prime \prime}$. This beam size represents the optimal combination between resolution and sensitivity for these particular WSRT observations and is the standard resolution HALOGAS used.

We compare these numbers with those derived for the SKA as shown in Table 1. For a $10^{h}$ observation with SKA1-MID using a $30^{\prime \prime}$ beam and $5 \mathrm{~km} \mathrm{~s}^{-1}$ velocity resolution, the resulting $5 \sigma$ column density limit is $2.9 \cdot 10^{18} \mathrm{~cm}^{-2}$. In other words, a $10^{h}$ SKA1-MID observation already goes twice as deep as HALOGAS. This therefore opens the prospect of routinely obtaining deep observations of a large number of galaxies in a modest amount of time, and efficiently characterizing the presence of clouds, thick H I disks and other extra-planar features. This will lead to a more complete statistical description of the importance and mechanisms for accretion.

With $100^{h}$ of observing on SKA1-MID (again at $30^{\prime \prime}$ and $5 \mathrm{~km} \mathrm{~s}^{-1}$ ), a column density limit of $9.3 \cdot 10^{17} \mathrm{~cm}^{-2}$ is reached. This is comparable to the column densities that deep H I observations of nearby galaxies with the Green Bank Telescope reach (Pisano 2014), but with a spatial resolutions that is almost a factor 20 better (from $9^{\prime}$ to $30^{\prime \prime}$ ).

A 50\% SKA1-MID will still be able to reach HALOGAS depth, but in $10^{h}$ per galaxy instead of $120^{h}$. In terms of increasing the statistics of accretion processes these observations will therefore already be scientifically valuable.

For a fixed observing setup, the column density sensitivity of SKA1-SUR is lower by a factor of 2.7 (for $3^{\prime \prime}$ ) to 6.4 (for $30^{\prime \prime}$ ). Though observations will thus be less sensitive, the large field of view will help exploring the extended environment around nearby galaxies, and can help placing the SKA1-MID results in the context of the large-scale cosmic web.

For the full SKA, at a resolution of $30^{\prime \prime}$, the column density limits after $10^{h}$ are truly impressive, reaching $\sim 2.8 \cdot 10^{17} \mathrm{~cm}^{-2}$, i.e., more than an order of magnitude deeper than HALOGAS. At $100^{h}$, these numbers go down even further to $\sim 8.9 \cdot 10^{16} \mathrm{~cm}^{-2}$. At these column densities one would be able to detect the 21-cm emission from Lyman Limit Systems. Further discussion on this and the cosmic web in general is presented in the chapter by Popping et al. (2015).

\section{Accretion and external high-velocity clouds}

\subsection{HVCs as accretion probes}

The previous section emphasised the importance of the extra-planar gas as a tracer of accretion. Here we discuss some of the properties of the clouds that form part of that extra-planar component. In our Milky Way (MW) these are observed as HVCs (see the chapter by McClure-Griffiths et al. 
2015 elsewhere in this volume). Here we discuss the prospects of observing these clouds in other galaxies.

The physical properties of the HVCs around the MW span a large range; the large complexes are at distances of $\sim 10 \mathrm{kpc}$, with similar heights above the Galactic plane, and have physical sizes of several kpc and masses of $1-50 \times 10^{5} M_{\odot}$ (Putman et al. 2012; Wakker et al. 2008, 2007). Compact clouds near the disk-halo interface are $\lesssim 1 \mathrm{kpc}$ above the disk and have sizes and masses of $\sim 700 M_{\odot}$ and $\sim 30 \mathrm{pc}$ (Ford et al. 2010). In addition, there may be populations of clouds distributed throughout the halo of the MW. These compact high velocity clouds (CHVCs) appear to be circumgalactic and at distances of $\sim 50-150 \mathrm{kpc}$ with masses of $\sim 5 \times 10^{4} M_{\odot}$ and sizes of $\sim 0.8 \mathrm{kpc}$ for an assumed distance of $100 \mathrm{kpc}$ (Sternberg et al. 2002; Maloney \& Putman 2003; Putman et al. 2002; de Heij et al. 2002). The difference in observed properties of the HVCs is a clue to their likely origins. The velocities of the large complexes and their low metallicities $\left(\sim 0.15 Z_{\odot}\right)$ are consistent with accretion of gas onto the MW (Wakker et al. 2007). The spatial distribution and kinematics of the clouds at the disk-halo interface are consistent with a galactic fountain (Ford et al. 2010). The same phenomenon is traced by the IVCs. The CHVCs potentially provide information on the Galactic dark matter substructure (Sternberg et al. 2002).

HVCs are thus a probe of both accretion in galaxies and the recycling of gas through the hot halo as part of the galactic fountain. Observing and understanding these processes in our own Galaxy is hampered by the difficulty of constraining the distance to these systems. In external galaxies, we can directly determine the masses and sizes of the HVCs and have the opportunity to view the HVCs in relation to the galactic disks. Unfortunately, attempts to search for HVCs in external galaxies are often hampered by lack of resolution and sensitivity. HVCs are low column density structures with peak column densities of $\sim 10^{19} \mathrm{~cm}^{-2}$. The compact clouds (disk-halo and CHVCs) will be point source detections for most extragalactic surveys with masses $<10^{5} M_{\odot}$ down to $\sim 100 M_{\odot}$ for clouds at the disk-halo interface.

Only a handful of nearby disk galaxies outside the Local Group have detections of discrete clouds of anomalous HI: NGC 891 with clouds of $1-3 \times 10^{6} M_{\odot}$ (Oosterloo et al. 2007), NGC 2403 with clouds of $6-10 \times 10^{6} M_{\odot}$ (Fraternali et al. 2001), NGC 2997 with clouds of $\sim 10^{7}$ $M_{\odot}$ (Hess et al. 2009) and NGC 55 with clouds of $2-40 \times 10^{6} M_{\odot}$ (Westmeier et al. 2013). These anomalous H I clouds appear to be the analogs of the large HVC complexes in the MW with substantial H I masses, large extents of several kpcs and projected separations from the galactic disks of $\sim 10-20 \mathrm{kpc}$. Other dedicated surveys of other nearby galaxies and galaxy groups reveal no HVC analogs down to $\sim 4 \times 10^{5} M_{\odot}$ at larger distances from galaxies (Pisano et al. 2007; Irwin et al. 2009).

Further systematic studies of nearby galaxies are thus needed to determine the properties of the global HVC population. Observations much deeper than those that currently exist are needed to extend the extragalactic HVC population to the low mass clouds at the disk-halo interface that are associated with the galactic fountain mechanism.

\subsection{Prospects for HVCs with the SKA}

Table 3 summarizes the properties of HVCs. The large HVC complexes have physical sizes of $\sim 10 \mathrm{kpc}$ and will remain resolved with a $30^{\prime \prime}$ beam out to distances of $\sim 30 \mathrm{Mpc}$, so observing requirements in the nearby Universe will be set by column density limits. For typical $z$-heights of 
Table 3: Galactic and Extragalactic HVCs

\begin{tabular}{|c|c|c|c|c|c|c|c|}
\hline Class & $d_{H I}(\mathrm{kpc})$ & $\begin{array}{c}\theta_{H I} \\
\left(D_{M p c}^{-1} \mathrm{kpc}\right)\end{array}$ & $N_{H I}\left(\mathrm{~cm}^{-2}\right)$ & $M_{H I}\left(M_{\odot}\right)$ & $z$-height (kpc) & $\begin{array}{c}\theta_{z} \\
D_{M p c}^{-1} \sin (i)\end{array}$ & References \\
\hline & (1) & $(2)$ & (3) & (4) & $(5)$ & (6) & \\
\hline $\mathrm{CHVCs}^{b}$ & 0.78 & $2.7^{\prime}$ & $1.4 \times 10^{19}$ & $5 \times 10^{4}$ & 100 & $5.7^{\circ}$ & Putman et al. (2002) \\
\hline Disk-halo clouds & 0.030 & $6^{\prime \prime}$ & $1 \times 10^{19}$ & 700 & 0.66 & $2.3^{\prime}$ & Ford et al. (2010) \\
\hline
\end{tabular}

$\sim 10 \mathrm{kpc}$, the HVC complexes should be spatially resolved from the disk out to $10 \mathrm{Mpc}$ for all but the most face-on systems $\left(i>20^{\circ}\right)$ at $30^{\prime \prime}$ resolution. In more face-on systems they can however be detected because of their velocity separation from the main disk (e.g., in M101; Kamphuis et al. 1991). In a $10^{h}$ observation, SKA1-MID can achieve a $5 \sigma$ column density sensitivity of $\sim 5 \times 10^{18}$ $\mathrm{cm}^{-2}$ in a $20 \mathrm{~km} \mathrm{~s}^{-1}$ channel (matched to the typical linewidth of the HVCs). This is a factor of 2 lower than the typical peak column density for MW HVCs; at this level a survey of nearby galaxies would be sensitive to the full range of MW HVC complexes rather that just the most massive systems with the highest peak column densities. The full SKA will offer greatly improved column density sensitivity. Increasing sensitivity to lower column density allows the tracing of the extended structure of the HVCs which can offer clues to the environment, including the pressure of the medium they are embedded in. Higher resolution observations trace the clumpiness and structure of the $\mathrm{H}$ I complexes.

The compact clouds at the disk-halo interface are physically very small and will be mostly unresolved point sources except at the highest resolutions in the closest galaxies. With a typical size of $30 \mathrm{pc}$ they have angular sizes of $6^{\prime \prime} D_{M p c}^{-1}$. For a $25 \mathrm{~km} \mathrm{~s}^{-1}$ channel (matched to the linewidth of HVCs; Table 2 scaled by velocity width), SKA1-MID with a $10^{\prime \prime}$ beam is sensitive to the typical disk-halo cloud of Ford et al. (2010) of $\sim 700 M_{\odot}$ at $1 \mathrm{Mpc}$ in a $100^{h}$ observation. Deep observations of very nearby galaxies $(D=2 \mathrm{Mpc}$ ) would be sensitive to the most massive disk-halo clouds $\left(M_{\mathrm{HI}}>3000 M_{\odot}\right)$. With a sensitivity improvement of $\sim 10$ for the full SKA, the median disk-halo cloud population will be detectable in deep $\left(100^{h}\right)$ observations out to $\sim 3 \mathrm{Mpc}$.

SKA1-SUR lacks the column density sensitivity to efficiently detect the large HVC complexes in large surveys; neither does it have the point source sensitivity to detect the disk-halo clouds. However, its large field of view does make it ideal for deep surveys of nearby galaxies to search for an extended circumgalactic HVC population separated from the parent galaxy by multiple degrees. With a $10 \mathrm{hr}$ observation at $30^{\prime \prime}$ and $20 \mathrm{~km} \mathrm{~s}^{-1}$, SKA1-SUR has a column density sensitivity of $\sim 4 \cdot 10^{19} \mathrm{~cm}^{-2}$. This would allow for the detection of the high- $N_{\mathrm{HI}}$ tail of the CHVC population. At $100^{h}$ the sensitivity is $\sim 1.2 \cdot 10^{19} \mathrm{~cm}^{-2}$; this is well-matched to the median peak $N_{\mathrm{HI}}$ of the CHVC population.

\section{Summary}

Two of the main unanswered questions in galaxy evolution are: "how do the astrophysics at the scales of individual clouds and gas complexes lead to global star formation laws?" and "where 
do galaxies acquire their gas from?" Much progress has been made on these questions in recent years, but the required combination of column density sensitivity and high spatial resolution that is needed to definitively answer these questions has so far been missing. For example, a proper study of the first problem has so far really only been possible in the MW and the nearest galaxies in the Local Group (see the chapter by McClure-Griffiths et al. 2015 elsewhere in this volume). Similarly, the second question has so far only been addressed for a limited number of galaxies with investment of a large amount of observing time. SKA will enable us to make great progress in both areas.

For studies of individual objects, SKA1-MID provides the highest resolution and sensitivity. With a maximum effective angular resolution of $3^{\prime \prime}$ SKA1-MID will go as deep as the highestresolution existing $\mathrm{H}$ I surveys $\left(\sim 3 \cdot 10^{20} \mathrm{~cm}^{-2}\right.$ at $5 \sigma$ and $5 \mathrm{~km} \mathrm{~s}^{-1}$ - this limit definition applies to all values quoted here) in the same observing time $\left(10^{h}\right.$ per galaxy), but at a resolution that is at least twice as good. SKA1-SUR with its 18 square degree field of view will be able to instantaneously map the environment around the target galaxies. While (for a fixed observing time), its resolution and sensitivity will be more limited, this will nevertheless provide invaluable information on the context of the observed objects.

The full SKA will go an order of magnitude deeper than SKA1-MID and SKA1-SUR. SKA will allow mapping of individual galaxies at $1^{\prime \prime}$. After $10^{h}$ it will reach column densities of $2.5 \cdot 10^{20}$ $\mathrm{cm}^{-2}$, while $100^{h}$ gives a $5 \sigma$ limit of $8 \cdot 10^{19}$. This allows mapping of galaxies out to $10 \mathrm{Mpc}$ at a spatial resolution that has so far only been possible in our Local Group.

SKA1-MID has an exquisite column density sensitivity, and at an angular resolution of $30^{\prime \prime}$ is extremely efficient in detecting low-column density H I. At $10^{h}$ it will reach limits of $2.9 \cdot 10^{18}$ $\mathrm{cm}^{-2}$, which is twice as deep as the deepest existing observations at comparable resolutions. A $100^{h}$ integration gives limits of $9.3 \cdot 10^{17} \mathrm{~cm}^{-2}$, comparable to what is achieved with the singledish Green Bank Telescope, but at 20 times better resolution. SKA1-SUR will again be able to provide quantitative information on the environment and larger surroundings of any detections.

The full SKA will be truly ground-breaking: after $10^{h}$ its column density limit at $30^{\prime \prime}$ angular resolution is $\sim 2.8 \cdot 10^{17} \mathrm{~cm}^{-2}$, after $100^{h}, \sim 8.9 \cdot 10^{16} \mathrm{~cm}^{-2}$. The latter will enable a direct exploration of the link between galaxies and the cosmic web, and should reveal accretion processes in great detail.

SKA will therefore revolutionize our understanding of star formation in other galaxies, the processes leading to it, as well as the ways by which galaxies acquire the necessary gas.

\section{References}

Bagetakos, I., Brinks, E., Walter, F., et al. 2011, AJ, 141, 23

Begum, A., Chengalur, J.N., Karachentsev, I.D., Sharina, M.E., Kaisin, S.S. 2008, MNRAS 386, 1667

Bigiel F., Leroy A., Walter F., Brinks E., de Blok W.J.G., Madore B., Thornley M.D., 2008, AJ, 136, 2846

Bigiel F. et al., 2011, ApJ, 730, L13

Blyth, S.-L., et al., 2015, "Exploring Neutral Hydrogen and Galaxy Evolution with the SKA", in proc. Advancing Astrophysics with the Square Kilometre Array, PoS(AASKA14)128 
Borthakur, S., Yun, M. S., \& Verdes-Montenegro, L. 2010, ApJ, 710, 385

Braun, R., Thilker, D. A., Walterbos, R. A. M., \& Corbelli, E. 2009, ApJ, 695, 937

Braun, R., 2014, SKA-TEL-SKO-DD-XXX, Rev A DRAFT 5 (2014-06-02)

Bregman, J. N. 1980, ApJ, 236, 577

Calzetti, D., Liu, G., \& Koda, J. 2012, ApJ, 752, 98

Calzetti, D. 2013, Secular Evolution of Galaxies, 419

Cannon, J. M., Giovanelli, R., Haynes, M. P., et al. 2011, ApJL, 739, L22

Chiappini, C., Matteucci, F., and Gratton, R. 1997, ApJ, 477, 765

Chomiuk, L. and Povich, M. S. 2011, AJ, 142, 197

de Blok, W.J.G., 2010, Advances in Astronomy, 2010, article id. 789293

de Blok, W. J. G. \& Walter, F. 2006, AJ, 131, 363

de Blok, W. J. G., Keating, K. M., Pisano, D. J., et al. 2014, arXiv:1407.3648

de Heij, V., Braun, R., and Burton, W.B. 2002, A\&A, 392, 417

Dewdney, P.E., et al., 2013, SKA-TEL-SKO-DD-001 (2013-03-13)

di Cintio, A., Brook, C., Dutton, A.A., Macciò, A.V., Stinson, G.S., Knebe, A. 2014, MNRAS, 441, 2986

Dutta, P., Begum, A., Bharadwaj, S., \& Chengalur, J. N. 2009, MNRAS, 398, 887

Dutta, P., Begum, A., Bharadwaj, S., \& Chengalur, J. N. 2013, New Astronomy, 19, 89

Fernandez, X., Joung, M. R., and Putman, M. E. 2012, ApJ, 749, 181

For, B.-Q., Koribalski, B.S., Jarrett, T.H. 2012, MNRAS 425, 1934

Ford, H.A., Lockman, F.J., and McClure-Griffiths, N.M. 2010, ApJ, 722, 367

Fraternali, F. and Binney, J. J. 2008, MNRAS, 386, 935

Fraternali, F. and Tomassetti, M. 2012, MNRAS, 426, 2166

Fraternali, F., Oosterloo, T., Sancisi, R., van Moorsel, G. 2001, ApJL, 562, L47

Fraternali, F., van Moorsel, G., Sancisi, R., and Oosterloo, T. 2002, AJ, 123, 3124

Fraternali, F. 2013, IAU Symp. 298 (arXiv:1310.2956)

Fukui, Y., Kawamura, A., Wong, T., et al. 2009, ApJ, 705, 144

Heald, G., Józsa, G., Serra, P., et al. 2011, A\&A, 526, A118

Heald, G. et al. 2015, A\&A, in prep

Hess, K.M., Pisano, D.J., Wilcots, E.M., and Chengalur, J.N., 2009, ApJ, 699, 762009.

Hopkins, A. M., McClure-Griffiths, N. M., and Gaensler, B. M. 2008, ApJ, 682, L13

Hunter, D. A., Ficut-Vicas, D., Ashley, T., et al. 2012, AJ, 144, 134

Ianjamasimanana, R., de Blok, W. J. G., Walter, F., \& Heald, G. H. 2012, AJ, 144, 96

Irwin, J.A, Hoffman, G.L., Spekkens, K., et al., 2009, ApJ, 692, 1447

Jütte, E., Heald, G., et al. 2015, A\&A, in prep.

Kamphuis, J., Sancisi, R., \& van der Hulst, T. 1991, A\&A, 244, L29

Karachentsev, I. D., Karachentseva, V. E., Huchtmeier, W. K., \& Makarov, D. I. 2004, AJ, 127, 2031

Karachentsev, I. D., Makarov, D. I., \& Kaisina, E. I. 2013, AJ, 145, 101

Kennicutt R. C., Jr., 1989, ApJ, 344, 685

Kennicutt R. C., Jr., 1998, ARA\&A, 36, 189

Kereš, D., Katz, N., Weinberg, D. H., \& Davé, R. 2005, MNRAS, 363, 2

Kim, S., Staveley-Smith, L., Dopita, M. A., et al. 2003, ApJS, 148, 473 
Koribalski, B. S. 2008, in "Galaxies in the Local Volume", Astrophysics and Space Science Proceedings, eds. Koribalski \& Jerjen, Springer Netherlands, p. 41

Leroy A. K., Walter F., Brinks E., Bigiel F., de Blok W. J. G., Madore B., Thornley M. D., 2008, AJ, 136, 2782

Maloney, P.R., and Putman, M.E., 2003, ApJ, 589, 270

Mapelli, M., Moore, B., \& Bland-Hawthorn, J. 2008, MNRAS, 388, 697

Marinacci, F., Binney, J., Fraternali, F., Nipoti, C., Ciotti, L., and Londrillo, 2010, MNRAS, 404, 1464

McClure-Griffiths, N.M., et al., 2015 "Galactic and Magellanic Evolution with the SKA", in proc. Advancing Astrophysics with the Square Kilometre Array, PoS(AASKA14)130

Mundell, C. G., James, P. A., Loiseau, N., Schinnerer, E., \& Forbes, D. A. 2004, ApJ, 614, 648

Nelson, D., Vogelsberger, M., Genel, S., Sijacki, D., Keres, D., Springel, V., Hernquist, L. 2013, MNRAS, 429, 3353

Norman, C. A., \& Ikeuchi, S. 1989, ApJ, 345, 372

Oh, S.-H., Brook, C., Governato, F., Brinks, E., Mayer, L., de Blok, W.J.G., Brooks, A., Walter, F. 2011, AJ, 142, 24

Oosterloo, T., Fraternali, F., \& Sancisi, R. 2007, AJ, 134, 1019

Ott, J., Wong, T., Pineda, J. L., et al. 2008, PASA, 25, 129

Ott, J., Stilp, A. M., Warren, S. R., et al. 2012, AJ, 144, 123

Panter, B., Jimenez, R., Heavens, A. F., \& Charlot, S. 2007, MNRAS, 378, 1550

Pisano, D. J., Barnes, D. G., Gibson, B. K., et al. 2007, ApJ, 662, 959

Pisano, D. J. 2014, AJ, 147, 48

Popping, A., et al., 2015, "Observations of the Intergalactic Medium and the Cosmic Web in the SKA era", in proc. Advancing Astrophysics with the Square Kilometre Array, PoS(AASKA14) 132

Putman, M. E., de Heij, V., Staveley-Smith, L., et al. 2002, AJ, 123, 873

Putman, M. E., Peek, J. E. G., \& Joung, M. R. 2012, ARA\&A, 50, 491

Roychowdhury, S., Chengalur, J. N., Kaisin, S. S., \& Karachentsev, I. D. 2014, MNRAS, 445, 1392 Ryan-Weber, E., et al. 2008, MNRAS 384, 535

Morganti, R., et al., 2015, "Cool Outflows and HI absorbers with SKA", in proc. Advancing Astrophysics with the Square Kilometre Array, PoS(AASKA14)134

Sancisi, R., \& Allen, R. J. 1979, A\&A, 74, 73

Sancisi, R., Fraternali, F., Oosterloo, T., \& van der Hulst, T. 2008, A\&A Rev, 15, 189

Schönrich, R. and Binney, J. 2009, MNRAS, 396, 203

Schruba A., et al., 2011, AJ, 142, 37

Serra, P., Oosterloo, T., Morganti, R., et al. 2012, MNRAS, 422, 1835

Shapiro, P. R., \& Field, G. B. 1976, ApJ, 205, 762

Stanimirović, S., Murray, C. E., Lee, M.-Y., Heiles, C., \& Miller, J. 2014, ApJ, 793, 132

A. Sternberg, C. F. McKee, and M. G. Wolfire 2002, ApJS, 143, 419

Stilp, A. M., Dalcanton, J. J., Warren, S. R., et al. 2013, ApJ, 765, 136

Swaters, R. A., Sancisi, R., and van der Hulst, J. M. 1997, ApJ, 491, 140

Thilker D. A., et al., 2007, ApJS, 173, 538

Tumlinson, J., Thom, C., Werk, J. K., et al. 2013, ApJ, 777, 59 
van der Hulst, J. M., van Albada, T. S., \& Sancisi, R. 2001, Gas and Galaxy Evolution, 240, 451 Verdes-Montenegro, L., Yun, M. S., Williams, B. A., et al. 2001, A\&A, 377, 812

Wakker, B. P., York, D. G., Howk, J. C., et al. 2007, ApJL, 670, L113

Wakker, B. P., York, D. G., Wilhelm, R., et al. 2008, ApJL, 672, 298

Walter, F., Brinks, E., de Blok, W. J. G., et al. 2008, AJ, 136, 2563

Warren, B. E., Jerjen, H., \& Koribalski, B. S. 2004, AJ, 128, 1152

Warren, S. R., Skillman, E. D., Stilp, A. M., et al. 2012, ApJ, 757, 84

Westmeier, T., Braun, R., and Thilker, D., 2005, A\&A, 436, 101

Westmeier, T., Braun, R., \& Koribalski, B. S. 2011, MNRAS, 410, 2217

Westmeier, T., Koribalski, B.S., and Braun, R. 2013, MNRAS, 434, 3511

Young, L. M. \& Lo, K. Y. 1996, ApJ, 462, 203

Young, L. M. \& Lo, K. Y. 1997, ApJ, 490, 710

Young, L. M., van Zee, L., Lo, K. Y., Dohm-Palmer, R. C., \& Beierle, M. E. 2003, ApJ, 592, 111 